\title{
IMPACT OF OMEGA -3 ON HEALING OF BUCCAL TRAUMATIC ULCER OF ALBINO RATS (HISTOLOGICAL AND IMMUNOHISTOLOGICAL STUDY)
}

\author{
Nesreen Nabil Abdelsalam* and Sally Hassan Aboubakr*
}

\begin{abstract}
Introduction: Ulcers are one of the most frequent diseases affecting the oral cavity. The aim of this study was to assess the impact of omega- 3 on healing of buccal traumatic ulcer in rat.

Materials and methods: In this study adult male rats in 2 groups ( $n=15$ per group) were used. A wound circular in shape (4 $\mathrm{mm}$ diameter and $2 \mathrm{~mm}$ depth) was punched into the buccal mucosa of each rat using biopsy punch. The control group was not treated, the study group was treated with $93 \mathrm{mg} / \mathrm{kg}$ body weight omega-3 that was guided to the stomach by gastric gavage. Histological samples were harvested on post-injury days 3,7 and 14 .
\end{abstract}

Results: The study group had high reepithelialization and connective tissue healing score on day 7 and 14 . The control group had high inflammation score on day 3 and 7, and low reepithelializtion score on day 3,7 and 14 .

Conclusion: Omega-3 compound increases fibroblast counts and decreases inflammatory cell counts, and also causes an increase in reepithelialization and epithelial thickness.

\section{INTRODUCTION}

The mucous membrane that lines the oral cavity consists of two layers: an outer layer of stratified squamous epithelium and an underlying layer of dense connective tissue (lamina propria). In those regions where there is looser connective tissue, beneath the lamina propria a submucosa exists containing blood vessels, fat and glands (eg, cheek, soft palate). The oral mucosa has both epithelial and connective tissue structural modifications in the different regions of the oral cavity ${ }^{(1)}$.
Ulcer can be the final common manifestation, often clinically indistinguishable, of a wide and complex spectrum of conditions including traumatic lesions, infectious, vesiculo-bullous, neoplastic and gastrointestinal diseases ${ }^{(2)}$. Their formation represents the final outcome of a complex and orchestrated phenomenon involving both epithelial lining and connective tissue, reduction of blood flow, cytokines production, cell death, wound repair, all participate to the dynamic process which was defined as ulcer ${ }^{(3)}$.

\footnotetext{
* Lecturer at Oral Biology Department, Faculty of Dentistry, Mansoura University
} 
Ulcer healing is an active process of filling the mucosal defects with proliferating and migrating epithelial and connective tissue cells. At the ulcer margin, epithelial cells proliferate and migrate on the granulation tissue to reepithelialze the ulcer and also invade granulation tissue to reconstruct glandular structures within the ulcer. The reepithelialization and reconstruction is controlled by several growth factors ${ }^{(4)}$.

The goal of treatment of oral ulcers is to relieve symptoms; therefore, finding suitable drugs with fewer side effects is the goal of many researchers, because of the side effects of chemically synthesized drugs, there is a trend toward the use of more natural treatments ${ }^{(5)}$.

$\mathrm{N}-3$ fatty acids, popularly referred to as $\mathrm{W}-3$ fatty acids or omega-3 fatty acids, are types of a series of essential unsaturated fatty acids that cannot be synthesized by the human body but are vital for normal metabolism, Omega-3 acids were found in almost all cases to be very effective in rapidly clearing infection and promoting healing ${ }^{(6)}$.

Omega-3 fatty acids in fish oil may have a beneficial effect on early wound epithelialization, but may inhibit later collagen deposition, possibly minimizing scar formation ${ }^{(7)}$.

The consumption of dietary fish oil result in altered lymphocytes and suppress the production of proinflammatory cytokines by macrophage, also supplementation of the diet with omega- 3 results in decreased monocytes, neutrophils and decreased the production of proinflammatory cytokines and interleukin-2 ${ }^{(8)}$.

Vascular endothelial growth factor (VEGF), also known as vascular permeability factor (VPF) is considered to be the most endothelial specific and most relevant of the known angiogenic growth factor ${ }^{(9)}$. VEGF is the most significant mediator of wound angiogenesis, and its production stimulates capillary growth to provide adequate nutrients, oxygen, and inflammatory cells. During wound, healing, capillary density reaches more than twice of uninjuried normal tissue ${ }^{(10)}$.

\section{MATERIALS AND METHODS}

Thirty adult male albino rats approximately $120-200 \mathrm{gm}$ in weight were used in this study. The animals were housed in individual cage and received standard diet for rodent and tap water.

\section{Animals were divided into 2 groups:}

\section{Group I:}

Consisted of 15 rats, which received corn oil (vehicle of the drug), $2 \mathrm{ml}$ each orally by gastric gavage in equal divided dose $/ 12 \mathrm{~h}$ after ulcer induction.

\section{Group II:}

Consisted of 15 rats, which received omega-3 fatty acid of $93 \mathrm{mg} / \mathrm{kg}$ body weight per day dissolved in corn oil orally by gastric gavage in equal divided dose $12 / \mathrm{h}$ after ulcer induction ${ }^{(11)}$.

\section{Methods:}

\section{General anesthesia:}

General anaethesia was performed by intravenous injection of sodium thiopental $15 \mathrm{mg} / \mathrm{kgm}$ body weight, anesthesia was maintained with etherane (Counta 7) 15mg/ml.

\section{Ulcer induction:}

The left buccal mucosa of animals were cleaned with chlorhexidine digluconate $1 \%$ in water. The field was dried; the traumatic ulcer was made opposite to the first molar, midway between mandible and maxilla. The buccal mucosa was bunched out using biopsy bunch to ensure that all ulcers having the same size, the ulcer was circular in shape about $4 \mathrm{~mm}$ in diameter and $2 \mathrm{~mm}$ in depth. 


\section{Biopsy collection:}

5 rats from each group were scarified at the days 3, 7 and 14 respectively. Rats were anesthetized then killed with over dosage of halothane by putting fully saturated cotton with halothane with the animal in a closed box. Then longitudinal specimen containing the traumatic ulcer was dissected out from each rat, each specimen was obtained and immediately fixed in $10 \%$ formalin solution.

\section{Histological analysis:}

The specimens were fixed in a fixative solution for 24 hours and processed to paraffin blocks. Routine sections was prepared at $4 \mu \mathrm{m}$ and stained with:

1. Heamatoxyline and eosin stain.

2. Immunohistochemical staining for vascular endothelial growth factor (VEGF Ab-1).

\section{HISTOLOGICAL FINDINGS}

\section{1- Haematoxylin and Eosin Stain:}

\section{Group I (induced traumatic ulcer without re- ceiving treatment):}

\section{Group I at 3 days:}

At 3 days; the sections showed slight proliferation of the covering epithelium and formation of the granulation tissue with dilated blood vessels, fibroblasts, collagen fibers and dense inflammatory cells infiltration (Fig 1).

\section{Group I at 7 days:}

At 7 days, the partial healing process appeared through the proliferation of the covering epithelium, the involving cellular connective tissue stoma appeared with large number of fibroblasts, collagen fibers, newly formed blood vessels and inflammatory cell infiltrations (Fig 2).

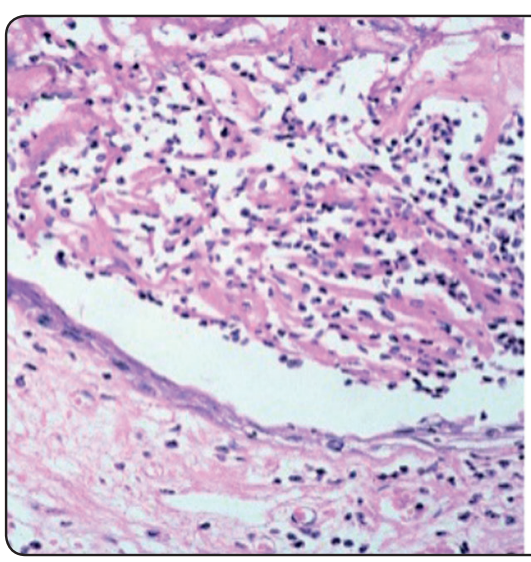

Fig. (1) Photomicrograph of the experimental group (I) at 3 days showing granulation tissue formation with heavy inflammatory cells infiltration, fibroblasts and collagen fibers ( $\mathrm{H} \&$ E stain, $\mathrm{x} 400)$.
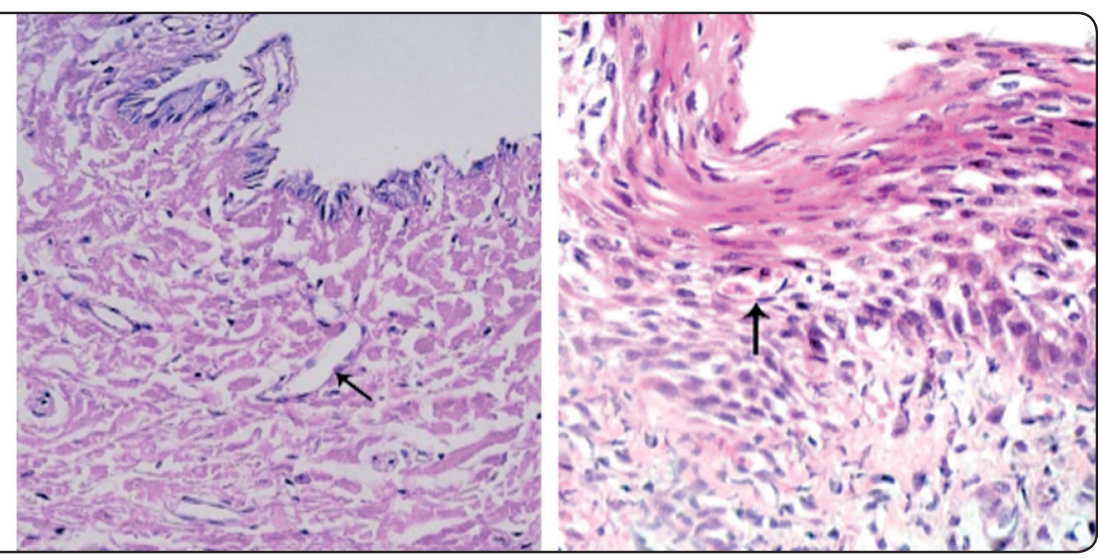

Fig. (2) Photomicrograph of the experimental group (I) at 7 days showing fibroblasts, initial organization of the collagen fibers and newly formed blood vessels (arrow) (H \& E stain, $\mathrm{x} 400)$.
Fig. (3) Photomicrograph of the experimental group (I) at 14 days showing reorganization of epithelium and connective tissue appear with fibroblasts, collagen fibers and blood vessels (arrow) (H \& E stain, $x 400)$. 


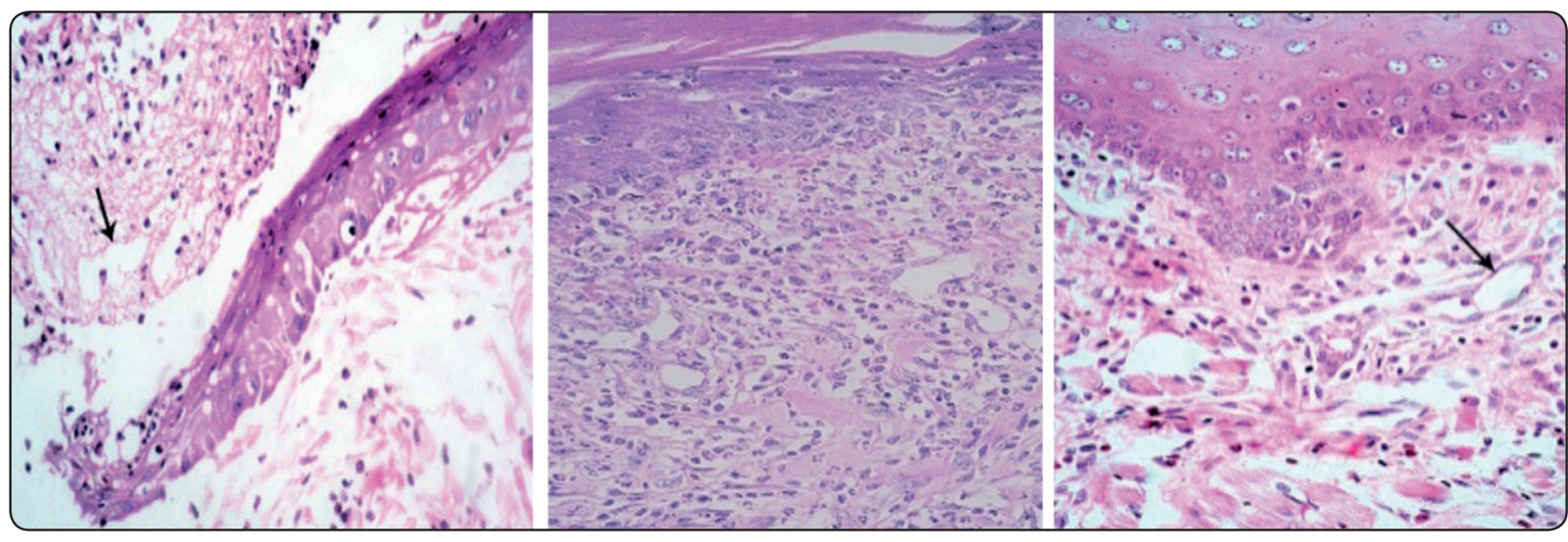

Fig (4): Photomicrograph of the ex- Fig (5): Photomicrograph of the ex- Fig (6): Photomicrograph of the experimental perimental group (II) at 3 days showing beginning of healperimental group (II) at 7 days group (II) at 14 days showing showing the connective tissue adequate epithelial stratification and ing of the epithelium and the granulation tissue appear with stroma with higher expression high cellular connective tissue stroma of fibroblasts, collagen bundles, few inflammatory cells fibroblasts, collagen fibers and blood vessels (arrow) (H \& E stain, $\mathrm{x} 400$ ). and wellformed blood vessels (H \& E stain, $\mathrm{x} 400$ ). with fibroblasts, collagen bundles, wellformed blood vessels (arrow) and few inflammatory cells (H \& E stain, $\mathrm{x} 400)$.

\section{Group I at 14 days:}

At 14 days; the sections showed marked epithelialization with obvious good architecture of connective tissue with blood vessels and decreased inflammatory cells infiltrations (Fig 3).

\section{Group II (induced traumatic ulcer recieving treatment)}

\section{Group II at 3 days:}

The sections showed the beginning of the healing process via proliferation of the epithelium. The granulation tissue appeared with fibroblasts, collagen fibers, inflammatory cells infiltrations and newly formed blood vessels. (Fig 4).

\section{Group II at 7 days:}

After 7 days, the healing process was evident as the covering stratified epithelium proliferated to close the ulcer margins and covered the underlying connective tissue stroma which formed from large number of fibroblasts, collagen bundles, newly formed blood vessels and decreased inflammatory cells infiltrations (Fig 5).

\section{Group II at 14 days:}

The advanced healing process appeared by prominent proliferation of the covering epithelium as well as the well-formed connective tissue stroma with fibroblasts, collagen bundles, wellformed blood vessels and few inflammatory cells (Fig 6).

\section{Immunohistochemical Results:}

VEGF (Lab Vision Ab-1) was used in this study to detect the presence of VEGF in the tissues, which has cellular localization in the cytoplasm, cell surface and connective tissue stroma.

VEGF expression was confirmed by the presence of positive brown-stained cytoplasm in the epithelial cells in the specimens. Staining of endothelial cells, fibroblasts and some infiltrating inflammatory cells was also observed ${ }^{(12)}$. 


\section{Group I (induced traumatic ulcer without re- ceiving treatment):}

\section{Group I at 3 days:}

There was increase expression of VEGF than previous group. The positive immune reaction appeared in the epithelium, endothelial cells, fibroblasts and inflammatory cells (Fig 7).

\section{Group I at 7 days:}

In this group, there was obvious expression of VEGF at the epithelium, in the fibroblasts, infiltrating inflammatory cells, muscle fibers and vascular endothelial cells of blood vessels (Fig 8).

\section{Group I at 14 days:}

After 14 days, the epithelium and connective tissue showed decrease expression of VEGF than the previous group with obvious reepithelialization and advanced healing (Fig 9).

\section{Group II (induced traumatic ulcer receiving treatment):}

\section{Group II at 3 days:}

There was increase expression of VEGF at epithelium, fibroblasts, infiltrating inflammatory cells and vascular endothelial cells of blood vessels at the granulation tissues (10).

\section{Group II at 7 days:}

There was obvious expression of VEGF at the epithelium, in the fibroblasts, some infiltrating inflammatory cells in the connective tissue and in the endothelial cells around blood vessels (Fig 11).

\section{Group II at 14 days:}

After 14 days of injury, the section showed obvious reepithelialization and advanced healing with decrease immunohistochemical reaction for VEGF at the epithelium and connective tissue than the previous group and parallel group (II) (Fig 12).

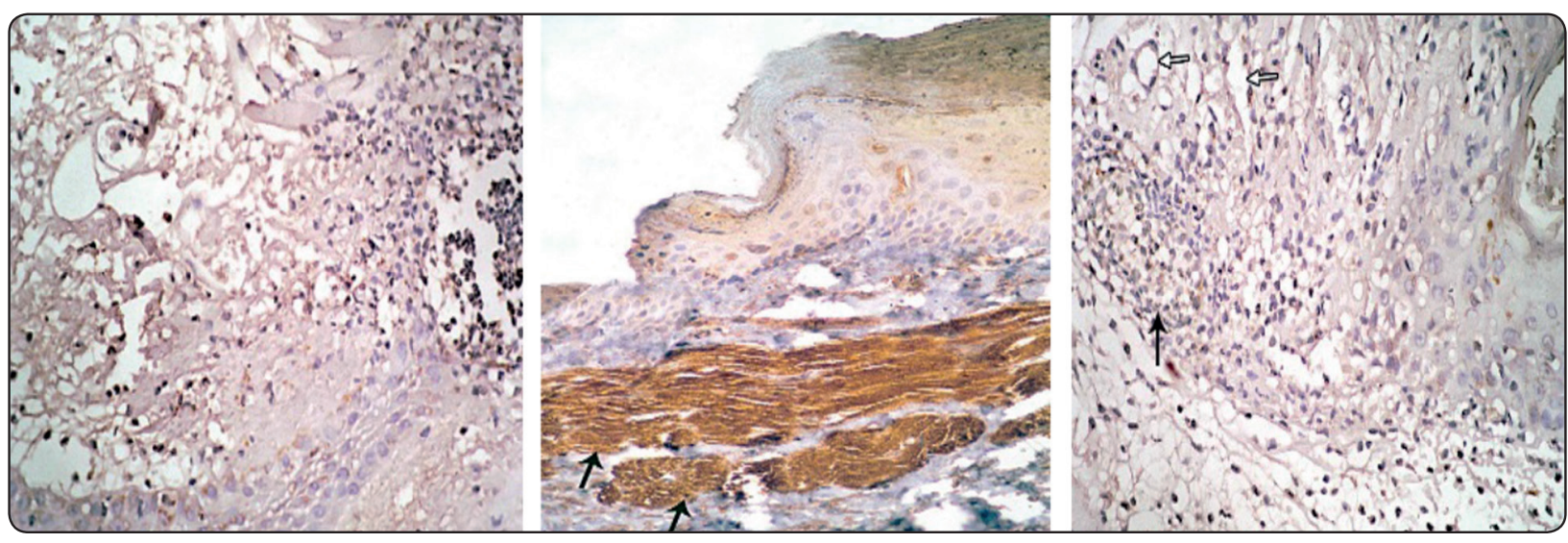

Fig. (7): Photomicrograph of the ex- Fig. (8): Photomicrograph of the ex- Fig (9): Photomicrograph of the experimental perimental group (I) at $3 \mathrm{rd}$ days showing increase expression of VEGF in endothelial cells, some fibroblasts and infiltrating inflammatory cells (arrow) (IHC staining, DAB chromogen $\mathrm{x} 400$ ) perimental group (I) at 7th days showing obvious expression of VEGF in epithelial cells, infiltrating inflammatory cells, fibroblasts and muscle fibers (arrows) (IHC staining, DAB chromogen $x$ 400). group (I) at 14 days showing decrease expression of VEGF at the epithelium and connective tissue stroma than previous group. The positive staining appear in endothelial cells of blood vessels (white arrow), fibroblasts and residual inflammatory cells (black arrow) (IHC staining, DAB chromogen x 400). 


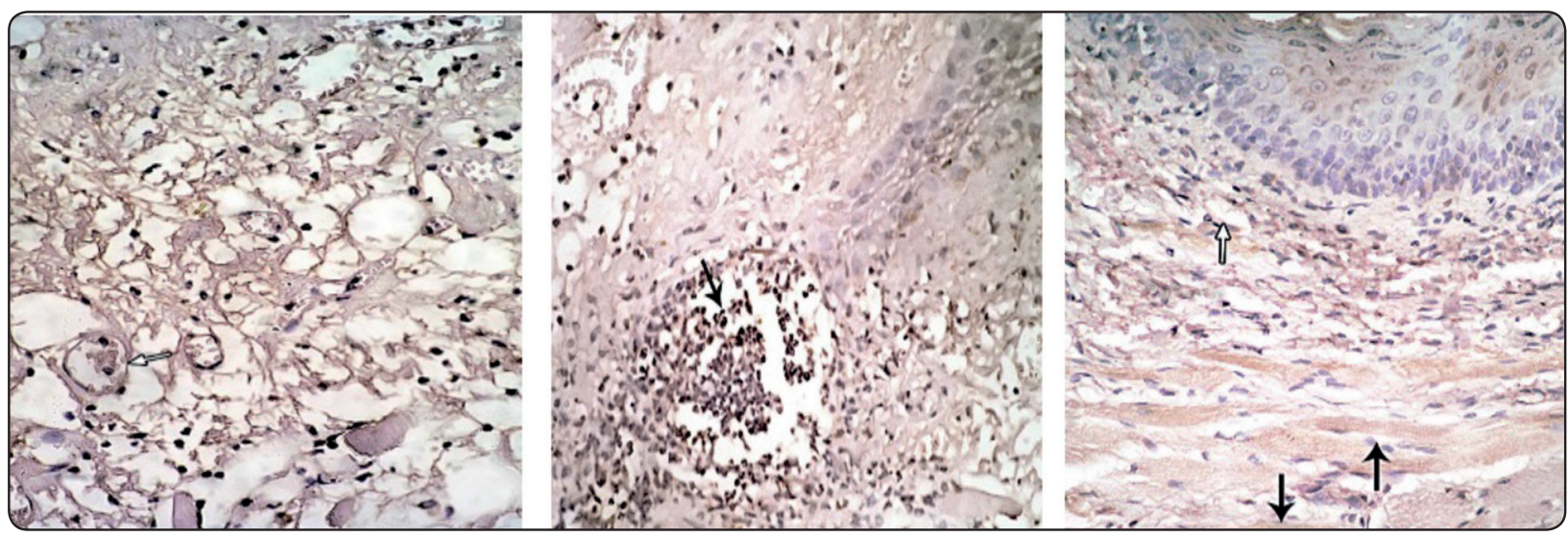

Fig (10): Photomicrograph of the Fig (11): Photomicrograph of the ex- Fig (12): Photomicrograph of the experimental experimental group (II) at perimental group (II) at 7 days, 3 days showing increase showing obvious staining of immune reaction at the VEGF in epithelial cells, fifibroblasts, inflammatory cells and endothelial cells of blood vessels (arrow) (IHC staining, DAB chromogen $\mathrm{x} 400$ ) broblasts, inflammatory cells (arrow)and endothelial cells of blood vessels (IHC staining, DAB chromogen $\mathrm{x} 400$ ). group (II) at 14 days showing decrease immune reaction of VEGF. The positive staining appear in fibroblasts, inflammatory cells, endothelial cells of blood vessels ( white arrow) and muscle fibers (black arrow) (IHC staining, DAB chromogen $\mathrm{x} 400$ ).

\section{DISCUSSION}

Ulcer may be ultimately considered as a wound, and its persistence in oral cavity depends on the equilibrium between pathologic noxae (chronic as well as temporary) and host tissue ability to put into effect the reparative process. Indeed, wound healing is a complex process involving collaborative efforts of multiple types of epithelial, dermal, immunity cells and cytokines such as soluble growth factors and interleukins ${ }^{(13)}$.

Vascular endothelial growth factor is considered the most endothelial specific and the most relevant of the known angiogenic growth factor ${ }^{(9)}$. But VEGF is unique for its effects on multiple components of the wound healing cascade, including angiogenesis and recently shown epithelialization and collagen deposition ${ }^{(14)}$.

There are many trials to improve healing of oral ulcers, but no specific treatment for ulceration was definitely documented. The primary goals of therapy for ulcers are relief of pain, reduction of ulcer duration, and restoration of normal oral function. Secondary goals include reduction in the frequency and severity of recurrences and maintenance of remission. Topical medications, such as antimicrobial mouth washes and topical corticosteroids, systemic medications can be tried if topical therapy is ineffective ${ }^{(15)}$.

Although several medical and palliative therapies are available, because of the importance and prevalence of oral ulcers, application of a new therapy that is less expensive, easy to use, and readily available would be helpful ${ }^{(5)}$.

A number of investigations have demonstrated that a diet supplemented with fish oil enriched in omega-3 fatty acids has profound beneficial health effects against various conditions, including cardiovascular diseases, respiratory diseases, diabetes, depression, cancers, inflammatory disease, and immunological renal disorders. Dietary omega-3 fatty acids have shown a significant protective effect against gastric ulcerations by inhibition of offensive mucosal factors and augmentation of defensive mucosal factors ${ }^{(16)}$. 
After 3 days, the ulcerative area of experimental group (I), revealed slight proliferation of the covering epithelium and the granulation tissue appeared with dilated blood vessels, fibroblasts, collagen fibers and dense inflammatory cells infiltration, while in the treated group (II), There was more granulation tissue formation with marked dilated blood vessels.

In agreement with our result, Arterburn etal., 2006 stated that Dietary supplements of $\omega-3$ polyunsaturated fatty acids (PUFA) eicosapentaenoic acid (EPA) and docosahexaenoic acid (DHA), obtained primarily from fish oil could affect local cellular and molecular activity, such as the gene expression of proinflammatory cytokines, during the inflammatory stage of healing and thus, subsequent stages ${ }^{(17)}$.

After 7 days, in group (I), the partial healing process appeared through the slight proliferation of the covering epithelium, the involving cellular connective stroma appeared with large number of fibroblasts, collagen fibers, newly formed blood vessels and inflammatory cells infiltration. Meanwhile the group (II), showed marked reepithelialization with complete healing of connective tissue defect with few inflammatory cells.

In accordance to our results, Mariam et al., $2012^{(5)}$ showed that reepithelialization occurred more quickly in the Local and Systemic treated groups with omega -3 than in the other groups. Moreover, the greatest value of epithelial thickness was observed in the L and S groups on days 6 and 8 , which was significantly different from the other groups

In Hematoxylin and Eosin results after 14 days, there was complete healing of the ulcerative area and the underlying connective tissue in group (II) as compared to group (I) which showed progression in the healing process.

In agreement with our results, Calder, 2002(8) showed that the rapid healing observed with an omega-3 dressing is noted. Wounds treated with omega-3 show less edema, less granular and mononuclear cell (MNC) infiltration, less necrosis, and better wound contractions. Omega-3 acids play a role in the prevention of inflammation.

In our study, immunohistochemical study of VEGF in normal epithelium was observed as positive brown stain in cytoplasm of epithelial cells and connective tissue stroma. Turley et al., (1998) ${ }^{(18)}$ stated that in normal tissues, expression of VEGF appeared in connective tissue stroma of all tissue, variable proportion of the plasma cells, fibroblasts and lymphocytes. Also Banks et al., (1998) ${ }^{(19)} \&$ Maruyama et al., (2007) ${ }^{(20)}$ indicated that VEGF is produced by many cell types that participate in wound healing: endothelial cells, fibroblasts, smooth muscle cells, platelets, neutophils, and macrophages.

After 3 days, there was increased expression of VEGF in experimental group(I), these results was supported by Nissen et al., (1998) ${ }^{(21)}$ who observed that, keratinocytes at the wound edge expressed elevation in VEGF as early as 1 day after injury and eventually in epidermal cells, which migrate to cover the defect. The expression for VEGF $m$ RNA reaches a peak after 2-3 days, coincident with a peak in vascular permeability, and levels remain elevated until epidermal coverage is complete.

In-group (II), after 3 days, there was increase expression of VEGF at epithelium, fibroblasts, infiltrating inflammatory cells and vascular endothelial cells of blood vessels at the granulation tissues.

Our results were supported by Mariam et al., $2012^{(5)}$ which observed that Omega-3 increased the MNC and fibroblast counts and decreased the PMN cell count. Moreover, this compound increased reepithelialization and epithelium thickness. The findings of the present study indicate omega-3 increases the formation of granulation tissue, epithelialization, and fibroblast action, and reduces the residual wound area and clinical wound size. 
Also Goldberg et al., 2007 (22) reported that Omega-3, in addition to its antibacterial activity, may clear infection by stimulating the activity of leukocytes. Cell culture studies have shown that omega-3 stimulates proliferation of lymphocytes and monocytes to release cytokines, which are activators of the immune response to infection ${ }^{(22)}$.

At 7 days, there was obvious reaction of VEGF at both groups at the epithelium, fibroblasts, inflammatory cells and vascular endothelial cells. The above results in accordance with Chu et al., (2008) (23) in fracture healing, at 1 week, a great goal of positive expression of VEGF mRNA and VEGF was noted in inflammatory cells, fibroblasts, and vascular endothelial cells. This results was also supported by Howard et al., (1997) ${ }^{(24)}$ that maximal VEGF $m$ RNA was found between 3 and 7 days after wounding, during the period of granulation tissue formation.

The time course of VEGF expression provides insight into the progression of wound healing. During the proliferative phase of repair which occurring approximately 3 to 7 days post-wounding, capillary growth and differentiation are at maximum. During this period, VEGF is up-regulated to promote the early stages of angiogenesis (vascular dilation, permeability, migration, and proliferation) ${ }^{(14)}$.

Our results supported the role of omega-3 in accelerating ulcer healing and increased release of VEGF from many cells during early stages of wound healing, these results were in accordance with Kazuki Nagai,et al., $2014{ }^{(25)}$ who observed that $\omega-3$ polyunsaturated fatty acids docosahexaenoic acid (DHA) has an effect of softening the cell membrane of the vessel wall and promoting blood circulation, These omega-3-acid ethyl esters suppress inflammatory cytokines and leucocyte chemotactic factors, thus suggesting that wound healing could have been promoted as a result.

In the present work, after 14 days, group (II) showed decrease expression of VEGF in the epithelium and connective tissue stroma than the parallel group (I), after evidenced reepithelialization and complete connective tissue healing.

In agreement to our results, Takada et al., $2004^{(26)}$ have shown omega-3 fatty acids play a role in reepithelialization of experimental skin, and in intestinal and stomach wounds in rat, rabbit, and dog models, also Mariam et al., 2012 (5) reported that omega- 3 increases the formation of granulation tissue, epithelialization, and fibroblast action, and reduces the residual wound area and clinical wound size.

So, in accordance to our results, all the previous works demonstrated the direct and indirect role of VEGF as a significant factor in wound healing immediately after injury. Also our results revealed that systemic treatment with omega-3 has evidenced effect in accelerate ulcer healing in oral mucosa and may be a clinical candidate for treating oral mucosal lesions.

\section{REFERENCES}

1. Tracey A. Winning and Granted Townsend: Oral mucosal embryology and histology. Clinics in dermatology J 2000; 18(5):499-511.

2. Nikitakis NG, Sauk JJ, Papanicolaou SI: the role of apoptosis in oral disease: mechanism; aberrations in neoplastic, autoimmune, infectious, and developmental disease; and therapeutic opportunities. Oral surg oral Med oral Pathol oral Radial Endol .2004; 97(4): 476-490.

3. Campisi G, Compilato D, Cirillo N, Clavarella D, Panzarella V Amato S, Lo Muzio L: Oral ulcers: three questions on their physiopathology. Minerva Stomatol 2007;56(5):293-302.

4. Tarnawski A: Molecular mechanisms of ulcer healing. Drug News Perspect 2000:13(3):158.

5. Maryam Alsadat Hashemipour, Amir Reza Ghasemi, Mahdi Ansari Dogaheh, Molok Torabi. Effects of Locally and Systemically Applied n-3 Fatty Acid on Oral Ulcer Recovery Process in Rats. WOUNDS. 2012;24(9):258-266.

6. Fetterman JW Jr, Zdanowicz MM. Therapeutic potential of $n-3$ polyunsaturated fatty acids in diseases. Am J Health Syst Pharm. 2009;66:1169-1179. 
7. J. Wesley Alexander, Dorothy M. Supp. Role of Arginine and Omega-3 Fatty Acids in Wound Healing and Infection. Adv Wound Care (New Rochelle). 2014; 3(11): 682-690.

8. Calder PC. Dietary modification of inflammation with lipids. Proc Nutr Soc. 2002;61:345-358

9. Ferrara N, Chen H, Davis Symth T, Gerber HP, Nguyen TN, Chisholm V: Vascular endothelial growth factor is essential for corpus luteum angiogenesis. Nat Med. 1998;4:336-340.

10. Szpaderska AM, Walsh CG, Steinberg MJ, Dipierto LA: Distinct patterns of angiogenesis in oral and skin wounds. J of dent Res 2005;84(4):309-314.

11. Kris-Etherton Penny M., Harris William S., Apple Lawrence J. Fish Consumption, Fish Oil, Omega-3 Fatty Acids, and Cardiovascular Disease. Circulation. 2002;106:2747.

12. Johnstone S, Logan RM: Expression of vascular endothelial growth factor in normal oral mucosa, oral dysplasia and oral squamous cell carcinoma. Int J Oral maxillofac Surg. 2007; 36: 263-6.

13. Campisi G, Compilato D, Cirillo N, Clavarella D, Panzarella V Amato S, Lo Muzio L: Oral ulcers: three questions on their physiopathology. Minerva Stomatol 2007;56(5):293-302.

14. Bao P, Kodra A, Tomic-Canic M, Golinko Ms, Ehrlich HP, Brem $\mathrm{H}$ : The role of vascular endothelial growth factor in wound healing. J Surg Res 2009;153:347-358.

15. Barrons RW: Treatment strategies for recurrent oral aphthous ulcers. Am J Health System Pharm 2001; 58(1):41-50.

16. Bhattacharya A, Ghosal S, Bhattacharya SK. Effect of fish oil on offensive and defensive factors in gastric ulceration in rats. Prostaglandins Leukot Essent Fatty Acids. 2006; 74:109-116.

17. Arterburn LM, Hall EB, Oken H. Distribution, interconversion, and dose response of $\mathrm{n}-3$ fatty acids in humans. Am J Clin Nutr. 2006;83 Suppl:1467S-1476S.
18. Turley H, Scott PA, Watts VM, Bicknell R, Harris AL, Gatter $\mathrm{KC}$ : Expression of VEGF in routinely fixed material using a new monoclonal antibody VG1. J Pathol 1998; 186: 313-318.

19. Banks RE, Forbes MA, Kinsey SE, Stanley A, Ingham E, Walters C, Selby P J: Release of the angiogenic cytokine vascular endothelial growth factor (VEGF) from platelets: significance for VEGF measurements and cancer biology. B r J Cancer 1998; 77: 956-964.

20. Maruyama K, Asai J, Li M, Thorne T, Losordo DW, DA more PA: Decreased macrophage number and activation lead to reduced lymphatic vessel formation and contribute to impaired diabetic wound healing. Am J Pathol 2007; 170: 1178-1191.

21. Nissen NN, Polverini PJ, Koch AE, Volin MV, Gamlli RL, Dipitro L A: Vascular endothelial growth factor mediates angiogenic activity during the proliferative phase of wound healing. Am J Pathol 1998;152: 1445-1445.

22. Goldberg RJ, Katz J. A meta-analysis of the analgesic effects of omega-3 polyunsaturated fatty acid supplementation for inflammatory joint pain. Pain. 2007;129(1-2):5-7.

23. Chu TW, Liu YG, Wang ZG, Zhu PF, Liu D: Vascular endothelial growth factor and its receptor expression during the process of fracture healing. Chin $\mathrm{j}$ traumatol. 2008; 11(3): 161-164.

24. Howard EW, Soo C, Hunter S et al; Differences in MMP and TIMP gene expression during wound repair. Surg Forum 1997; 48: 688.

25. Kazuki Nagai, Katsuhiko Matsumaru, Ikuko Hirai, Yujiro Takae, and Kazuo Andoh. New Therapy Using Omega3-Acid Ethyl Esters for Decubitus Ulcers and Stasis Dermatitis: A Case Report. Iran Red Crescent Med J. 2014;16(12): e19500

26. Takada H, Takahash M, Soma, R, Kuwayama H. 3 polyunsaturated fatty acid and ulcer healing.J Med Sci. 2004;31:217-223. 